\title{
Advanced Methodologies for Manipulating Nanoscale Features in Focused Ion Beam
}

*Correspondence to:

Ahn JP,

Tel: +82-2-958-5536

Fax: +82-2-958-6974

E-mail: jpahn@kist.re.kr

Received December 3, 2015

Revised December 10, 2015

Accepted December 10, 2015

\author{
Yang-Hee Kim, Jong-Hyun Seo, Ji Yeong Lee, Jae-Pyoung Ahn* \\ Advanced Analysis Center, Korea Institute of Science and Technology (KIST), Seoul 02792, Korea
}

\begin{abstract}
Nanomanipulators installed in focused ion beam (FIB), which is used in the lift-out of lamella when preparing transmission electron microscopy specimens, have recently been employed for electrical resistance measurements, tensile and compression tests, and in situ reactions. During the pick-up process of a single nanowire (NW), there are crucial problems such as $\mathrm{Pt}, \mathrm{C}$ and $\mathrm{Ga}$ contaminations, damage by ion beam, and adhesion force by electrostatic attraction and residual solvent. On the other hand, many empirical techniques should be considered for successful pick-up process, because NWs have the diverse size, shape, and angle on the growth substrate. The most important one in the insitu precedence, therefore, is to select the optimum pick-up process of a single NW. Here we provide the advanced methodologies when manipulating NWs for in-situ mechanical and electrical measurements in FIB.
\end{abstract}

Key Words: Focused ion beam, Nanomanipulator, Nanowire, Rotation tip, Gripper

\section{INTRODUCTION}

Focused ion beam (FIB) has been used in diverse fields, including nanopatterning, transmission electron microscopy (TEM) specimen fabrication and cross-sectional observation, due to its unique high-precision milling using an ion beam with high-resolution scanning electron microscopy (SEM) observation using an electron beam (Giannuzzi et al., 1998). In addition to basic functions of FIB, nanomanipulators employed in FIB have supported various in-situ experiments which are to evaluate the electrical, mechanical, and chemical characteristics of nanomaterials in real time. For examples, the reaction mechanism of lithium ion battery is studied through a real-time lithium reaction test (Chou et al., 2015; Jung et al., 2015; Seo et al., 2015), mechanical characteristics of nanowires (NWs) are clearly revealed through a real-time tensile and compression tests (Seo et al., 2011; Seo et al., 2013), and electrical characteristics of nano features are investigated by directly electrical measurements (Roh et al., 2012; Yoon et al., 2012; Yoon et al., 2013).
For the in-situ experiments, one should firstly consider the pick-up process of NWs using the nanomanipulator. Several problems can be expected in the procedure. In general, NWs consists of the bundle type when the density is high and it makes the individual separation of NWs hard. We try to disperse the NWs in solution, but encounter another problem induced by the electrostatic adhesion of NWs and a substrate. Pt contamination is also a big issue for keeping a clean surface of NWs from FIB Pt deposition. As noted earlier, the structurally safe and compositionally clean sample preparation of a single NW is most important before proceeding the in-situ tests. On the other hand, three-dimensional (3D) understanding on the $\mathrm{X}, \mathrm{Y}$, and $\mathrm{Z}$ movements of nanomanipulators in FIB chamber is required. In this study, we used diverse nano tools such as nanotips, gripper, and rotation tip, being connected to nanomanipulator and proposed practical methodologies to selectively pick-up a single NW to evaluate the in-situ mechanical, electrical, and chemical characteristics of NWs, which have various growth directions, sizes, and degrees of dispersion.

@ This is an open-access article distributed under the terms of the Creative Commons Attribution Non-Commercial License (http://creativecommons.org/licenses/by-nc/4.0) which permits unrestricted noncommercial use, distribution, and reproduction in any medium, provided the original work is properly cited.

Copyrights @ 2015 by Korean Society of Microscopy 


\section{MATERIALS AND METHODS}

We adopted various kinds of NWs in this study, InAs NWs that was vertically grown on substrate by chemical vapor deposition (CVD) method, $\mathrm{CuInGaSe}_{2} \mathrm{NWs}$ grown from sapphire substrate, $\mathrm{Au}$ and $\mathrm{Ag}$ NWs dispersed in isopropyl alcohol, and carbon nanofibers synthesized by electrospinning. Controlling the motion at the nanoscale, we used the nanomanipulator (MM3A EM; Kleindiek Nanotechnik GmbH, Germany) as shown in Fig. 1A. The nanomanipulators of DCG Systems Inc. (USA) and Imina Technologies SA (Switzerland) were also proposed in Fig. $1 \mathrm{~B}$ and $\mathrm{C}$. These manipulators were installed inside a dual FIB (Quanta 3D; FEI, Netherlands) system. The practical contact with samples is conducted by nanotips that are connected at the end of nanomanipulators. In this study, a basic nonotip (Fig. 1D), showing a tungsten tip of $100 \mathrm{~nm}$ in diameter (PT-14-605-B; GGB Industries Inc., USA), was used with two special gripper in Fig. 1E (Ro-tip; Kleindiek Nanotechnik GmbH) and rotation tip in Fig. 1F (Kleindiek Nanotechnik $\mathrm{GmbH}$ ) for showing noble nanomanipulations. The manipulator is controlled with an accuracy of $0.25 \mathrm{~nm}$ by piezo motor. The rotation tip has an accuracy of $0.1^{\circ}$ and was capable of unlimited rotation in $360^{\circ}$ (Travel $360^{\circ}$ unlimited). The gripper has an accuracy of $20 \mathrm{~nm}$ and could express gripping force from a minimum of $5 \mu \mathrm{N}$ to a maximum of $5,000 \mu \mathrm{N}$. In the FIB chamber where the overall test was performed, a high-degree vacuum condition of approximately 2.54E-5 mbar was maintained and images were observed through an electron beam of $5 \mathrm{kV}$ and $24 \mathrm{pA}$.
A Cu Mesh Grid (G150-Cu; Electron Microscopy Sciences, USA), laser-diced (Laser workstation M2000; ALLTEC $\mathrm{GmbH}$, Germany) silicon wafer, and SEM-compatible glue (SEM Glue; Kleindiek Nanotechnik GmbH) were used for the in-situ sample preparation. Especially, the SEM glue is an epoxy-based material; this is hardened by polymerization in less than two minutes when it is exposed an accelerated electron beam over polymerization $(30 \mathrm{kV}, 0.35 \mathrm{nA})$. It has a strong bonding strength of approximately $2 \mathrm{mN}$.

\section{RESULTS AND DISCUSSION}

Fig. 2 shows SEM images of InAs, CuInGaSe 2 , and Au NWs synthesized by various methods. Those are to show difficult cases to pick up a single NW. In general, NWs manufactured by CVD methods have a vertical growth and low population that is relatively easy to pick up. As shown in Fig. 2A, however, it is hard to pick up a single NW from the NWs of high density. Fig. 2B shows CuInGaSe ${ }_{2}$ NWs grown from the sapphire substrate, which were grown with tilting angle of approximately $45^{\circ}$. Although they are relatively easy to pick up due to low density, the tilted angle disturbs the alignment of NW and nanotip. Fig. 2C shows Au NWs which are drop and dried on Si wafer from a liquid state dispersed in isopropyl alcohol. Although NWs in liquid are most commercially available, they have the disadvantage of a difficult pick-up. In particular, the pick-up process becomes even harder when NWs are in the bundled state in liquid solution and moved on Si wafer. Hence, it is important to check whether NWs can be dispersed well through simple stirring or sonication when
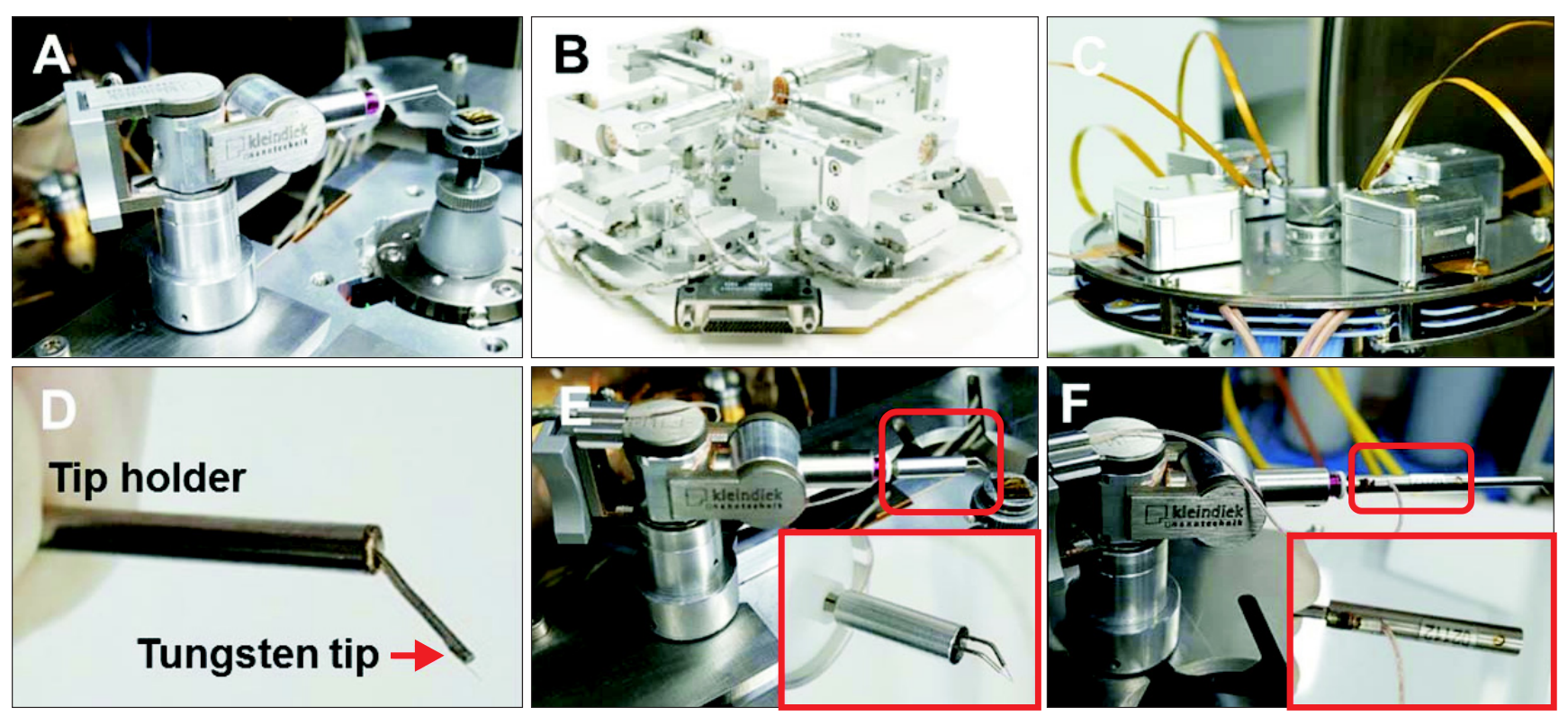

Fig. 1. Nanomanipulators made by Kleindiek, DCG Systems Inc., and Imina Technologies and special nanotips. Nanomanipulator of MM3A EM model (A), S100 model (DCG Systems Inc.) (B), PN14-4B-V model (Imina Technologies SA) (C). (D-F) Nanotips of nanotip, gripper, and ro-tip. 

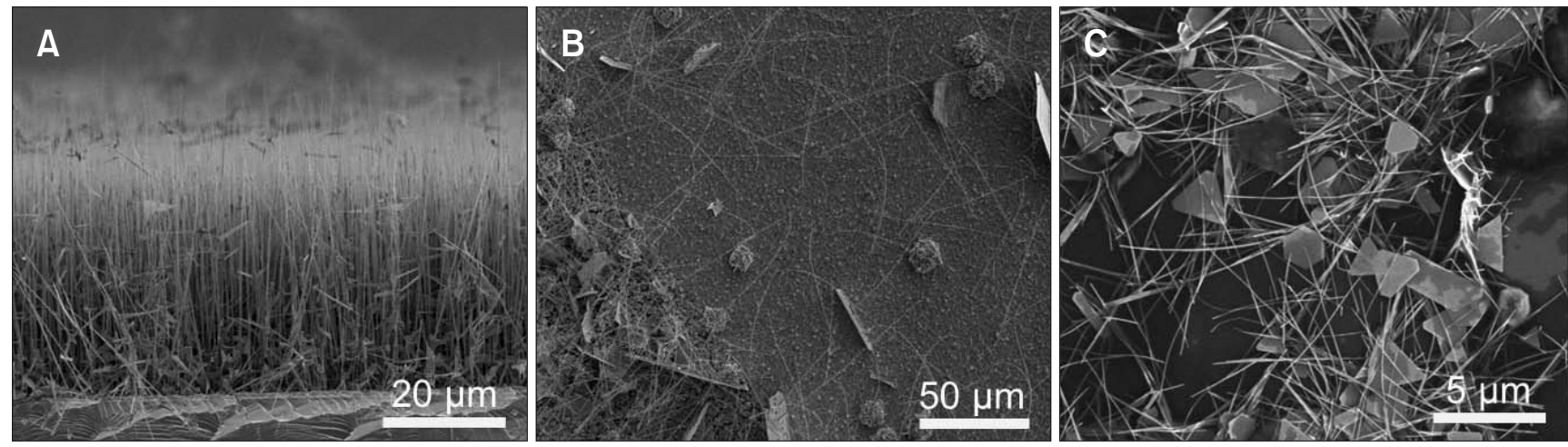

Fig. 2. Scanning electron microscopy images of nanowires (NWs) with diverse geometries and population. (A) InAs NWs vertically grown from a substrate. (B) $\mathrm{CuInGaSe}_{2}$ NWs grown from sapphire substrate with a tilted angle of $45^{\circ}$. (C) Bundled Au NWs after dropped and dried isopropyl alcohol including Au NWs on Si substrate.
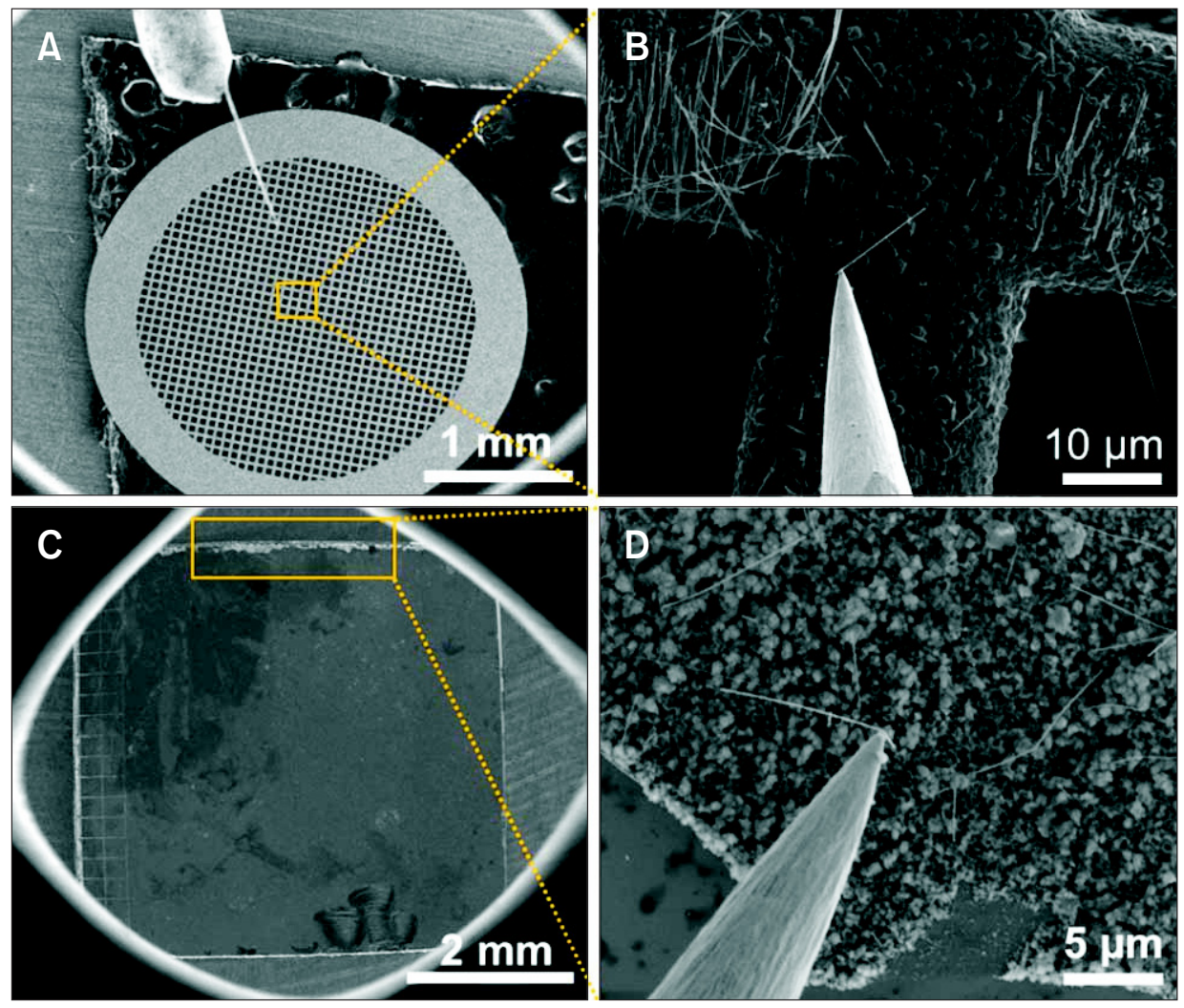

Fig. 3. Scanning electron microscopy images showing two pick-up processes. (A) A Cu mesh grid after slightly rubbing with a substrate that nanowires (NWs) are grown. (B) A magnified image of the Cu mesh grid where vertically grown InAs NWs are attached due to electrostatic attraction. (C) A laser-diced Si substrate. (D) Si debris around the edge of the laser-diced Si substrate where the liquid droplet including Ag NWs are dropped for pick-up.

purchasing NWs.

In case of having dense NW population as shown in Fig. $2 \mathrm{~A}$ and $\mathrm{C}$, we can consider two pick-up processes in Fig. 3. Although NWs vertically grown from a substrate are relatively easy to pick up compared to those in liquid, it becomes difficult when NWs are in bundles or tangled states. In this case, if the Cu TEM grid directly contacts the substrate and slightly rub together, the pick-up process becomes easier by the transfer of several NWs from substrate to $\mathrm{Cu}$ grid due to electrostatic attraction (Fig. 3A and B). On the other hand, NWs in liquid are frequently picked up after dropping and drying the liquid droplet onto Si substrate to remove the solvent. In this case, one decisive problem occur by the adhesion of NWs and the substrate due to electrostatic force or residual solvent. The NWs cannot be detached from the substrate well. Moreover, in the case of using a hard substrate, the tip of the manipulator can be damaged and curved during the pick-up process. In this case, a laser-diced Si wafer can be introduced to easily solve these problems. As shown in Fig. 3C and D, the laser diced Si substrate has Si debris around the 
edge, providing easy pick-up condition by prohibiting a close adhesion of NWs and the substrate after dropping and drying the liquid droplet, as shown in Fig. 3D.

Fig. 4 shows two fixation processes of thick nanofiber with sub-micrometer scale of about $400 \mathrm{~nm}$ in diameter. When picking up NWs, we often use the electrostatically attractive force but it is not sufficient to detach NWs which are strongly combined with the substrate. Therefore we usually use the Pt deposition method in FIB. In this case, however, we used to find $\mathrm{Ga}^{+}$contamination in the specimen and the sample damage due to the radiation of $\mathrm{Ga}^{+}$ion beam, even though a big change in SEM image isn't shown (Schilling et al., 2007). Both can occur crucial problems in not only TEM analysis but also in-situ mechanical and chemical tests. In order to escape such problems, therefore, we introduce the attachment method of a single NW and the nanotip by electron beam, which minimize the Pt contamination and doesn't damage the specimen. The amount of the Pt deposition needs to be experimentally at least 1.5 to 2 times thicker than the NW diameter to surely fix the NW on the tip in Fig. 4A. If the diameter of the NW is thicker than more $400 \mathrm{~nm}$, the deposition time over 20 minutes is more required. If not, the Pt junction can't be fixed well as shown in Fig. 4B.

In this case, we can introduce a new SEM glue hardened by the radiation of electron beam, which can be strongly and rapidly fixed within approximately $1 \mathrm{~min}$ without $\mathrm{Pt}$ and $\mathrm{Ga}^{+}$contaminations (Fig. 4C and D). Furthermore, it has high viscosity and low wettability so it doesn't easily evaporate inside the high-degree vacuum chamber of electron microscopy. The junction made by the SEM glue is very strong by hardening process of polymer. The hardening rate depends on the current density of nA level within several minutes. The SEM glue applications help aligning the NW and nanotip.

It is important to achieve one dimensional alignment of the loading direction and samples for the in-situ mechanical tensile and compressive tests. In many cases, however, the achievement of a good alignment is not easy due to two dimensional observations. If the picked sample can be rotated in Fig. 5A-D, we are able to check the angle. For example, the NW in Fig. 5A seems to have a good alignment but is not under the satisfactory alignment. If we perform the tensile test with misalignment, it leads to wrong results by introducing a large shear stress to the NWs. At this case, we can easily control the axis direction of specimen and manipulator tip using a rotation tip (Hoffmann et al., 2007).

For the bulk specimen like as an extremely large size in Fig. 5E-H, we cannot control the specimen using the general pickup method as mentioned before. The gripper is useful to pick the specimen easy and quick without an additional Pt-welding
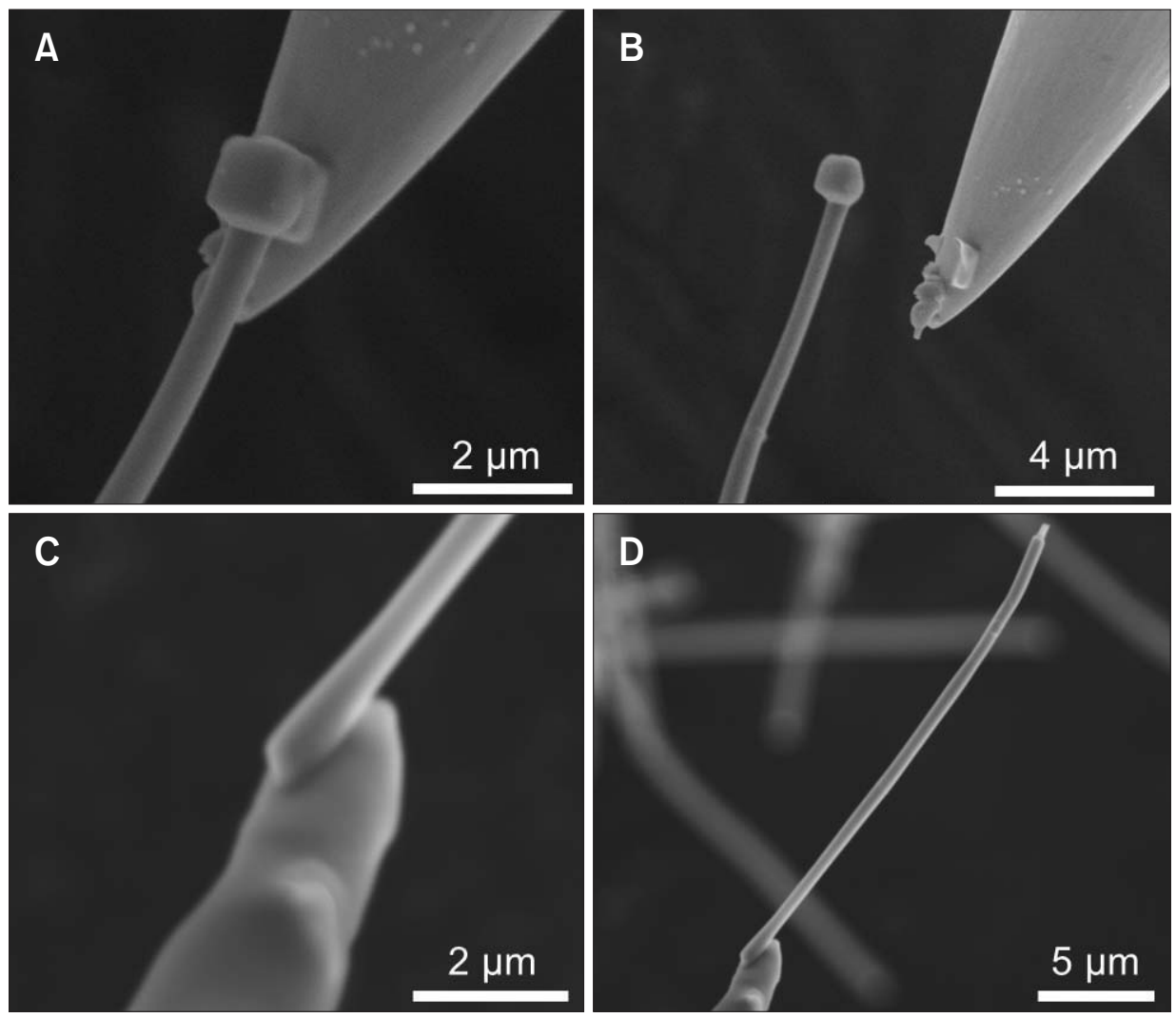

Fig. 4. The fixation procedure of a single nanowire (NW) by focused ion beam Pt deposition and scanning electron microscopy (SEM) glue. (A) Fixation of NW and nanotip by Pt deposition. (B) A failure of Pt junction weaker than the bonding strength of NWs and the substrate. (C) A new bonding method by an electron beam hardening SEM glue, which is hardened under an electron beam current of $1.5 \mathrm{nA}$ for 1 minute. (D) A successful pick-up image using SEM glue. 

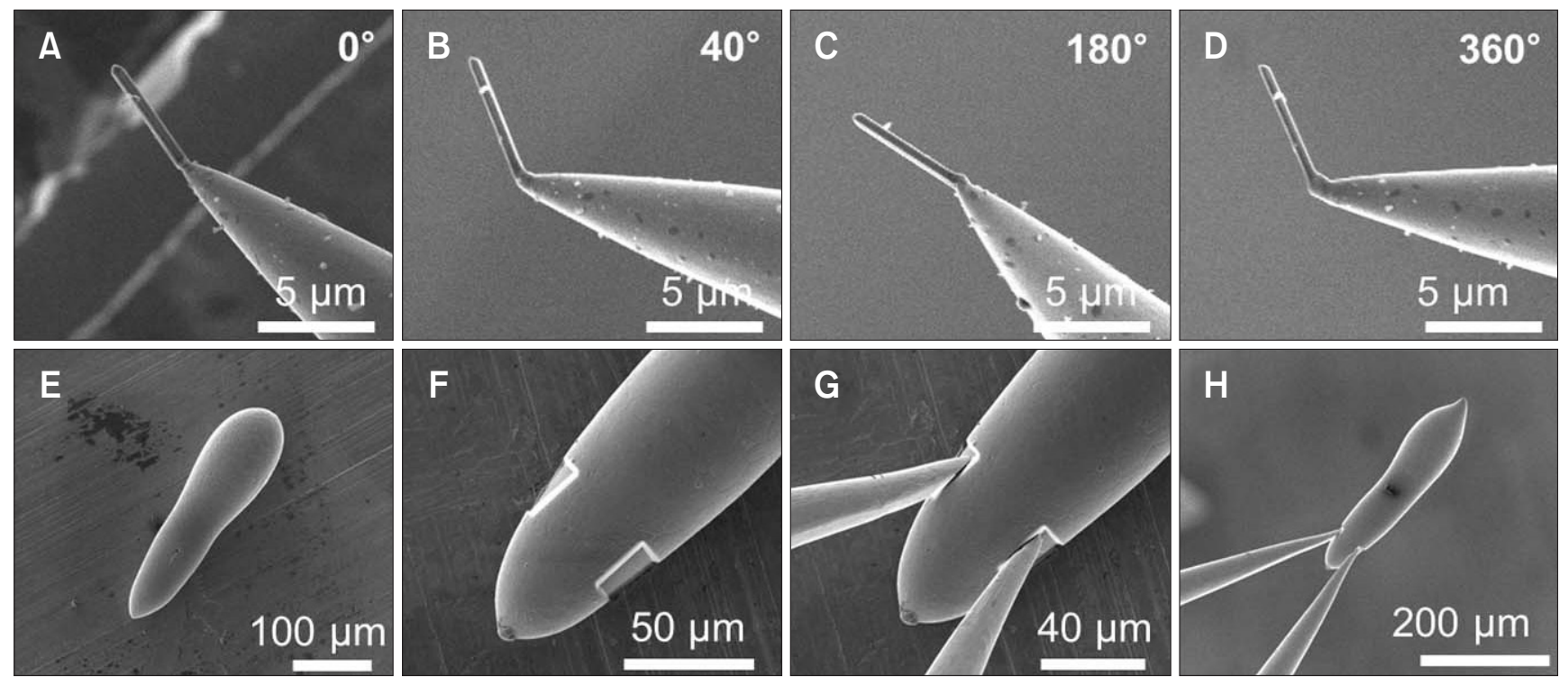

Fig. 5. Applications of the rotation tip and gripper. (A-D) A nanowire picked with any angle, which can be aligned by a rotation tip. (E-H) A lifting-out process by a gripper.

process or the $\mathrm{Ga}^{+}$and carbon contamination (Kristian et al., 2006). If the sample thickness is too small less than $100 \mathrm{~nm}$ to grip, this method is inconvenience for handling because the nanomaterials are attached to the gripper. Therefore the gripper method should be used over $100 \mathrm{~nm}$ of size.

\section{CONCLUSIONS}

For manipulating NWs for in-situ mechanical and electrical tests in FIB, we have reviewed several manipulation techniques in this work. As a common procedure, the diverse manipulation techniques could be summarized by three steps: 1) selecting a single NW, 2) aligning the NW and nanotip parallel to loading direction, and 3) strongly welding the junction between the NW and nanotip. For reducing the adhesion of the NWs and substrate, we have introduced substrates such as a TEM $\mathrm{Cu}$ grid and a laser-diced $\mathrm{Si}$ wafer for providing many edges and roughness. The contaminations originated from Pt deposition and Ga ion beam were a crucial problem for revealing NWs own characters in the in-situ tests. In order to overcome the problems, the SEM glue was used as a new connecting material in SEM and FIB chambers, which is hardened by the radiation of electron beam (about $1 \mathrm{nA}$ ) without the Pt and the Ga ion beam contamination. In addition, the rotation tip and gripper were also useful for controlling alignment and moving large-sized samples. For the in-situ sample preparation in FIB, on the other hand, we need to understand moving parts in 3D space for the good alignment between the nanotip and samples and then select a pick-up method to minimize the contamination by selecting the optimum pick-up process, and secure the junction with a high strength.

\section{CONFLICT OF INTEREST}

No potential conflict of interest relevant to this article was reported.

\section{REFERENCES}

Chou C Y, Seo J H, Hao Y H, Ahn J P, Paek E, Cho M H, Choi I S, and Hwang G S (2015) Anomalous stagewise lithiation of gold-coated silicon nanowires: a combined in situ characterization and firstprinciples study. ACS Appl. Mater. Interfaces. 7, 46976-16983.

Giannuzzi L A, Drown J L, Brown S R, Irwin R B, and Stevie F A (1998) Applications of the FIB lift-out technique for TEM specimen preparation. Microscopy Res. Tech. 41, 285-290.

Hoffmann S, Ostlund F, Michler J, Fan H J, Zacharias M, Christiansen S H, and Ballif C (2007) Fracture strength and Young's modulus of ZnO nanowires. Nanotechnology 18, 205503.

Jung M S, Seo J H, Moon M W, Choi J W, Joo Y C, and Choi I S (2015) A bendable Li- ion battery with a nano-hairy electrode direct integration scheme on the polymer substrate. Adv. Energy Mater. 5, 1400611.

Kristian M, Thomas W, Axel K, and Peter B (2006) Pick-and-place nanomanipulation using microfabricated grippers. Nanotechnology 17, 2434-2441. 
Roh J H, You Y H, Ahn J P, and Hwang J (2012) Electrical characterization of electronic materials using FIB-assisted nanomanipulators. Appl. Micro. 4, 223-227.

Schilling A, Adams T, Bowman R M, and Gregg J M (2007) Strategies for gallium removal after focused ion beam patterning of ferroelectric oxide nanostructures. Nanotechnology 18, 035301.

Seo J H, Chou C Y, Tsai Y H, Cho Y, Seong T Y, Lee W J, Cho M H, Ahn J P, Hwang G S, and Choi I S (2015) Ultrafast chemical lithiation of single crystalline silicon nanowires: in situ characterization and first principles modeling. RSC Adv. 5, 17438-17443.

Seo J H, Park H S, Yoo Y, Seong TY, Li J, Ahn J P, Kim B, and Choi I S (2013) Origin of size dependency in coherent-twin-propagation-mediated tensile deformation of noble metal nanowires. Nano Lett. 13, 5112-
5116.

Seo J H, Yoo Y, Park N Y, Yoon S W, Lee H, Han S, Lee S W, Seong T Y, Lee S C, Lee K B, Cha P R, Park H S, Kim B S, and Ahn J P (2011) Superplastic deformation of defect-free Au nanowires via coherent twin propagation. Nano Lett. 11, 3499-3502.

Yoon H, Kim S, Lee S, In J, Kim J, Ryoo H, Noh J H, Ahn J P, Jo Y, Choo J, and Kim B (2013) Three-dimensionally kinked high-conduction CoGe nanowire growth induced by rotational twinning. J. Mater. Chem. C 1, 6259-6264.

Yoon S W, Seo J H, Seong T Y, Kwon H, Lee K B, and Ahn J P (2012) Effects of Pt junction on electrical transport of individual $\mathrm{ZnO}$ nanorod device fabricated by focused ion beam. J. Nanosci. Nanotech. 12, 1466-1470. 